\title{
Estruturas Urbanas Comparadas: Estados Unidos e Brasil
}

Ricardo Machado Ruiz

Professor da Faculdade de Ciências Econômicas (FACE) e do Centro de

Desenvolvimento e Planejamento Regional (CEDEPLAR) da

Universidade Federal de Minas Gerais (UFMG)

\section{RESUMO}

Na literatura internacional são comuns afirmações de que os países em desenvolvimento têm estruturas urbanas extremamente concentradas e desiguais, enquanto que os países desenvolvidos apresentam uma rede urbana mais equilibrada. Este artigo compara as estruturas urbanas nacionais de dois países: um desenvolvido, os Estados Unidos da América, e outro em desenvolvimento, o Brasil.

\section{PALAVRAS-CHAVE}

estruturas urbanas, primazia, Lei de Zipf, distribuição de Pareto, economia urbana, economia regional

\section{ABSTRACT}

The literature on urban economics usually point out the developing countries as those ones with urban structure extremely concentrated and unequal, and the developed countries as those countries with a better distribution of cities. This paper compares the national urban structures of two countries: the USA, a developed country, and Brazil, a developing country.

KEY WORDS urban structure, primacy, Zipf's Law, Pareto distribution, urban economy, regional economy JEL Classification

$R / I, R / 2$ 


\section{COMPARANDO ESTRUTURAS URBANAS}

Em economia, assim como em outras áreas do conhecimento, as distribuições que descrevem populaçôes exibem uma peculiar regularidade entre a escala do evento e sua posição no ranking. Por exemplo, na economia a distribuição de renda o tamanho de cidades e firmas e o preço de ações podem ser representados por uma relação log-linear entre tamanho e posição no ranking. O mesmo ocorre em outras áreas, como, por exemplo, com os tamanhos de partículas de areia, os impactos dos meteoros na superfície lunar, a freqüência das palavras em textos e até mesmo o padrão das queimadas em florestas. Para cada um desses casos existem explicações, muitas delas polêmicas, para a emergência dessas regularidades. ${ }^{2}$

Na economia urbana, quando as cidades são ordenadas de forma decrescente a partir de sua população, há uma relação peculiar entre população e o ranking das cidades; em termos formais:

$$
\begin{aligned}
& \mathrm{R}_{\mathrm{i}}=\alpha / \mathrm{N}_{\mathrm{i}}^{\beta} \\
& \operatorname{Ln} \mathrm{R}_{\mathrm{i}}=(\operatorname{Ln} \alpha)-\beta\left(\operatorname{Ln} \mathrm{N}_{\mathrm{i}}\right)
\end{aligned}
$$

$\mathrm{R}_{\mathrm{i}}$ : rank da cidade $\mathrm{i}$

$\mathrm{N}_{\mathrm{i}}$ : população da cidade $\mathrm{i}$

$\alpha$ : constante.

Uma estrutura urbana com cidades extremamente assimétricas (cidades com diferentes tamanhos) e polarizadas (poucas grandes cidades) tenderia a ter um coeficiente $\beta<1$, enquanto que um país com cidades simétricas (cidades de portes similares) e estrutura urbana descentralizada (vários grandes centros urbanos) teria $\beta>1$. Quando $\beta=1$, temos um caso especial da chamada distribuição de Pareto: a Lei de Zipf.

Para se gerar uma distribuição Zipf em economia urbana uma hipótese é fundamental: todas as cidades devem possuir o mesmo potencial de crescimento, pouco importa seu tamanho ou posição nos seus espaços econômicos. Assim, caso as cidades tenham as mesmas chances de crescer, uma distribuição Zipf emergiria após um período de tempo. Entretanto, se algumas cidades forem mais ou menos favorecidas, a distribuição de tamanho se afastará da distribuição idealizada por Zipf, ou seja, $\beta \neq 1$. $^{3}$

Quando $\beta>1$, as cidades médias e pequenas são maiores que a ideal e/ou as cidades grandes têm deseconomias de aglomeração que limitam seu tamanho. Mas quando o oposto

1 Esta pesquisa teve o apoio financeiro da Pró-Reitoria de Pós-Graduação da Universidade Federal de Minas Gerais (PRPG-UFMG) e da Fundação de Amparo à Pesquisa do Estado de Minas Gerais (FAPEMIG).

2 Ver Alperovich (1984), Carol (1982), Gabaix e Ioannides (2003), Reed (2001), Richardson (1973).

3 No decorrer do texto o adjetivo "ideal" terá sempre como referência a distribuição de Zipf, onde $\beta=1$. Esta adjetivação não indica uma distribuição "ótima" de cidades. A definição de uma "distribuição ótima" requisitaria uma outra discussão teórica, tema este que está além do escopo do trabalho aqui proposto. 
se verifica $(\beta<1)$, são as grandes cidades as que se apresentam superdimensionadas; neste caso, as economias de aglomeração são superiores aos efeitos negativos presentes em grandes conurbações urbanas. Portanto, divergências em relação a $\beta=1$ podem indicar oportunidades de crescimento diferenciadas entre os núcleos urbanos, um resultado de economias de aglomeração e desaglomeração não proporcionalmente distribuídas. ${ }^{4}$

O debate sobre a distribuição do tamanho das cidades é extenso. Para vários casos, tais como França, Arábia Saudita, China, Israel e África do Sul, o coeficiente de Pareto tem apresentado recorrentes mudanças e valores diferentes do proposto pela Lei de Zipf. ${ }^{5}$ Rosen e Resnick (1980) estimam o coeficiente de Pareto para 44 países e encontram um coeficiente médio $\beta=1,14$ com um desvio padrão de 0,19 (Tabela 1 ), e em um estudo mais recente, para 73 países, Soo (2005) estima um coeficiente médio $\beta=1,11$ com um desvio padrão de 0,20 (Tabela 2). Estes estudos observam que existem claras indicações de não-linearidade (ou distorções) para uma grande parte das estruturas urbanas (33 países no trabalho de Rosen e Resnick e 53 países no estudo de Soo) e ressaltam que o coeficiente de Pareto é muito sensível à definição de cidade e do tamanho da amostra, o que traz dúvidas sobre a relevância da Lei de Zipf. Entretanto, ainda concluem que a regra tamanho-rank é uma ótima descrição das estruturas urbanas.

Parr (1985) propóe ainda que o coeficiente de Pareto teria, para cada país, uma trajetória histórica particular. No início do processo de urbanização existiria um vasto número de cidades relativamente simétricas $(\beta>1)$. Com a progressiva urbanização e industrialização surgiria uma estrutura urbana polarizada em torno de poucos centros urbanos $(\beta<1)$. Com o desenvolvimento do país haveria então uma nova desconcentração, explicada pela difusão das estruturas econômicas no espaço $(\beta>1)$. Parr (1985) especula que esse processo explicaria, por exemplo, as razóes para um baixo valor de $\beta$ (concentração urbana) nos países em desenvolvimento e um elevado $\beta$ para os países desenvolvidos (desconcentração urbana). ${ }^{6}$

Considerando a renda per capita de cada país como critério para mensuração do grau de desenvolvimento, e dividindo a amostra de Soo (2005) em duas, tem-se, para os países menos desenvolvidos, um coeficiente médio de Pareto $\beta=1,03 \mathrm{com}$ um desvio padrão de 0,16, enquanto que para os países mais desenvolvidos estas mesmas medidas seriam $\beta=1,29$ e 0,17 (Tabela 2). Em Rosen e Resnick (1980) essa maior polarização em países menos desenvolvidos é também registrada: $\beta=1,08$ para os menos desenvolvidos e $\beta=1,23$ para os mais desenvolvidos (Tabela 1 ). Em suma, esses 117 casos atestam a relevância dos argumentos de Parr (1985).

4 Para mais detalhes sobre essa hipótese, ver Gabaix (1999) e Gabaix e Ioannides (2003). Uma discussão sumária desse tema está em Fujita, Krugman e Venables (1999, capítulo 12).

5 Estudos de Song e Zhang (2002), Krakover (1998), Shukri e Alshuwaikhat (1996), Guerin-Pace (1995) e Naude e Krugell (2003) ilustram os casos citados.

6 Cuberes (2004) também sustenta esse movimento pendular (polarização-despolarização) como uma descrição acurada dos processos de urbanização e apresenta um modelo com essas características. 
TABELA 1 - COEFICIENTE DE PARETO, PRIMAZIA E RENDA PER CAPITA (US\$)

\begin{tabular}{|c|c|c|c|c|c|}
\hline Países & Ano & $\beta$ & Primazia 5 & Primazia 10 & Renda \\
\hline Suíça & 1970 & 1.10 & 0.38 & 0.18 & 35491 \\
\hline Dinamarca & 1971 & 1.37 & 0.56 & 0.22 & 23464 \\
\hline Japão & 1970 & 1.29 & 0.50 & 0.24 & 20465 \\
\hline Suécia & 1970 & 1.41 & 0.44 & 0.17 & 19269 \\
\hline Alemanha Ocidental & 1970 & 1.17 & 0.31 & 0.11 & 18392 \\
\hline Países Baixos & 1970 & 1.27 & 0.33 & 0.14 & 17189 \\
\hline E.U.A. & 1970 & 1.18 & 0.45 & 0.19 & 16893 \\
\hline França & 1970 & 1.33 & 0.55 & 0.24 & 16412 \\
\hline Áustria & 1971 & 0.88 & 0.70 & 0.50 & 15861 \\
\hline Normandia & 1970 & 1.27 & 0.55 & 0.25 & 15677 \\
\hline Finlândia & 1970 & 1.08 & 0.51 & 0.24 & 14980 \\
\hline Austrália & 1970 & 1.96 & 0.53 & 0.14 & 13861 \\
\hline Canadá & 1971 & 1.13 & 0.36 & 0.13 & 12568 \\
\hline Reino Unido & 1971 & 1.18 & 0.71 & 0.36 & 11858 \\
\hline Itália & 1971 & 1.05 & 0.36 & 0.18 & 10730 \\
\hline Israel & 1972 & 0.98 & 0.35 & 0.17 & 8690 \\
\hline Espanha & 1970 & 1.13 & 0.48 & 0.24 & 8417 \\
\hline Grécia & 1971 & 1.14 & 0.53 & 0.26 & 7487 \\
\hline Média Países Desenvolvidos & & 1.22 & 0.48 & 0.22 & 15984 \\
\hline Desvio Padrão & & 0.22 & 0.11 & 0.09 & 6325 \\
\hline Argentina & 1970 & 0.93 & 0.53 & 0.23 & 6823 \\
\hline Tchecoslováquia & 1970 & 1.11 & 0.51 & 0.26 & 5065 \\
\hline Venezuela & 1971 & 1.11 & 0.39 & 0.17 & 4306 \\
\hline África do Sul & 1970 & 1.00 & 0.23 & 0.10 & 4100 \\
\hline Hungria & 1970 & 1.09 & 0.76 & 0.45 & 2753 \\
\hline Brasil & 1970 & 1.15 & 0.44 & 0.22 & 2395 \\
\hline México & 1970 & 1.15 & 0.49 & 0.23 & 2295 \\
\hline U.R.S.S. & 1970 & 1.28 & 0.47 & 0.16 & 2049 \\
\hline Irã & 1966 & 0.99 & 0.64 & 0.36 & 1928 \\
\hline Turquia & 1970 & 1.08 & 0.49 & 0.26 & 1654 \\
\hline Alemanha Oriental & 1970 & 1.13 & 0.40 & 0.18 & 1491 \\
\hline lugoslávia & 1971 & 1.19 & 0.36 & 0.16 & 1491 \\
\hline Polônia & 1970 & 1.13 & 0.36 & 0.14 & 1491 \\
\hline Romênia & 1970 & 1.09 & 0.66 & 0.28 & 1491 \\
\hline Colômbia & 1973 & 0.85 & 0.48 & 0.29 & 1377 \\
\hline Malásia & 1970 & 0.97 & 0.37 & 0.17 & 1371 \\
\hline Filipinas & 1970 & 1.25 & 0.43 & 0.18 & 867 \\
\hline Marrocos & 1971 & 0.81 & 0.54 & 0.31 & 849 \\
\hline Tailândia & 1970 & 0.96 & 0.69 & 0.48 & 752 \\
\hline Zaire & 1970 & 0.93 & 0.52 & 0.29 & 546 \\
\hline Gana & 1970 & 1.10 & 0.55 & 0.30 & 475 \\
\hline Indonésia & 1971 & 0.97 & 0.53 & 0.29 & 298 \\
\hline Sri Lanka & 1971 & 1.13 & 0.55 & 0.26 & 285 \\
\hline Nigéria & 1963 & 1.54 & 0.31 & 0.10 & 264 \\
\hline Índia & 1971 & 1.20 & 0.36 & 0.16 & 213 \\
\hline Etiópia & 1971 & 0.97 & 0.69 & 0.44 & 106 \\
\hline Média Países em Desenvolvimento & & 1.08 & 0.49 & 0.25 & 1797 \\
\hline Desvio Padrão & & 0.15 & 0.13 & 0.10 & 1621 \\
\hline
\end{tabular}

Fonte: Elaboração própria a partir de Rosen e Resnick (1980) e World Bank (2003). 
TABELA 2 - COEFICIENTE DE PARETO E RENDA PER CAPITA (US\$)

\begin{tabular}{|c|c|c|c|c|c|}
\hline Países & Ano & $\begin{array}{l}\text { Número } \\
\text { Cidades }\end{array}$ & Coef. $\beta$ & & Renda \\
\hline Japão & 1995 & 221 & 1.317 & ** & 40830 \\
\hline Suíça & 1998 & 117 & 1.437 & ** & 40820 \\
\hline EUA & 2000 & 667 & 1.378 & ** & 34370 \\
\hline Noruega & 1999 & 41 & 1.270 & ** & 33630 \\
\hline Dinamarca & 1999 & 58 & 1.361 & ** & 32250 \\
\hline Suécia & 1998 & 120 & 1.439 & ** & 27660 \\
\hline Áustria & 1998 & 70 & 0.988 & & 26690 \\
\hline Alemanha & 1998 & 190 & 1.238 & ** & 26630 \\
\hline Países Baixos & 1999 & 97 & 1.473 & ** & 25270 \\
\hline Bélgica & 2000 & 68 & 1.590 & ** & 25070 \\
\hline Finlândia & 1999 & 49 & 1.192 & ** & 24750 \\
\hline França & 1999 & 104 & 1.451 & ** & 24460 \\
\hline Austrália & 1998 & 131 & 1.228 & ** & 21240 \\
\hline Itália & 1999 & 228 & 1.381 & ** & 20340 \\
\hline Canadá & 1996 & 93 & 1.245 & ** & 19910 \\
\hline Reino Unido & 1991 & 232 & 1.401 & ** & 16840 \\
\hline Israel & 1997 & 55 & 1.089 & * & 16710 \\
\hline Espanha & 1998 & 157 & 1.186 & ** & 14880 \\
\hline Portugal & 2001 & 70 & 1.382 & ** & 10900 \\
\hline Coréia do Sul & 1995 & 71 & 0.907 & ** & 10220 \\
\hline Média Países Densenvolvidos & & & 1.298 & & 24674 \\
\hline Desvio Padrão & & & 0.169 & & 8716 \\
\hline Grécia & 1991 & 43 & 1.413 & ** & 8560 \\
\hline Arábia Saudita & 1992 & 48 & 0.782 & ** & 7890 \\
\hline Argentina & 1999 & 111 & 1.044 & & 7550 \\
\hline República Tcheca & 2001 & 64 & 1.168 & ** & 5310 \\
\hline México & 2000 & 162 & 0.973 & & 5100 \\
\hline Chile & 1999 & 67 & 0.867 & ** & 4730 \\
\hline Hungria & 1999 & 60 & 1.124 & ** & 4620 \\
\hline Croácia & 2001 & 24 & 0.921 & & 4550 \\
\hline Venezuela & 2000 & 91 & 1.063 & * & 4310 \\
\hline Eslováquia & 1998 & 42 & 1.303 & ** & 4000 \\
\hline Polônia & 1998 & 180 & 1.183 & ** & 3860 \\
\hline Brasil & 2000 & 411 & 1.134 & ** & 3630 \\
\hline Turquia & 1997 & 126 & 1.054 & & 3180 \\
\hline África do Sul & 1991 & 94 & 1.360 & ** & 3050 \\
\hline Malásia & 1991 & 52 & 0.872 & * & 2540 \\
\hline Colômbia & 1999 & 111 & 0.902 & ** & 2150 \\
\hline Tailândia & 2000 & 97 & 1.186 & ** & 2020 \\
\hline Rússia & 1999 & 165 & 1.186 & ** & 1750 \\
\hline Belarus & 1998 & 41 & 0.844 & ** & 1560 \\
\hline
\end{tabular}

* Significante a $5 \%$ para $\beta \neq 1$ e ** Significante a $1 \%$ para $\beta \neq 1$

Fonte: Elaboração própria a partir de Soo (2005) e World Bank (2003). 
TABELA 2 - COEFICIENTE DE PARETO E RENDA PER CAPITA (US\$) (cont.)

\begin{tabular}{|c|c|c|c|c|c|}
\hline Países & Ano & $\begin{array}{l}\text { Número } \\
\text { Cidades }\end{array}$ & Coef. $\beta$ & & Renda \\
\hline Argélia & 1998 & 62 & 1.351 & ** & 1560 \\
\hline Irâ & 1996 & 119 & 1.058 & ** & 1510 \\
\hline Jordânia & 1994 & 34 & 0.898 & ** & 1470 \\
\hline Equador & 1995 & 42 & 0.808 & ** & 1440 \\
\hline Romênia & 1997 & 70 & 1.109 & * & 1430 \\
\hline Paraguai & 1992 & 19 & 1.014 & & 1420 \\
\hline Guatemala & 1994 & 13 & 0.729 & $* *$ & 1270 \\
\hline Casaquistão & 1999 & 33 & 0.962 & & 1260 \\
\hline Bulgária & 1997 & 23 & 1.114 & & 1200 \\
\hline Rep. Dominicana & 1993 & 23 & 0.847 & & 1180 \\
\hline Marrocos & 1994 & 59 & 0.874 & $\star *$ & 1170 \\
\hline Egito & 1996 & 127 & 0.996 & & 1100 \\
\hline Filipinas & 2000 & 87 & 1.080 & & 1020 \\
\hline Síria & 1994 & 10 & 0.744 & * & 980 \\
\hline lugoslávia & 1999 & 60 & 1.183 & * & 930 \\
\hline Ucrânia & 1998 & 103 & 1.025 & & 850 \\
\hline Indonésia & 1990 & 235 & 1.135 & ** & 620 \\
\hline Uzbekistão & 1997 & 17 & 1.049 & & 560 \\
\hline Paquistão & 1998 & 136 & 0.962 & & 470 \\
\hline Azerbaijão & 1997 & 39 & 1.035 & & 410 \\
\hline Quênia & 1989 & 27 & 0.817 & ** & 400 \\
\hline Índia & 1991 & 309 & 1.188 & ** & 350 \\
\hline China & 1990 & 349 & 1.181 & ** & 320 \\
\hline Sudão & 1993 & 26 & 0.909 & & 290 \\
\hline Bangladesh & 1991 & 79 & 1.091 & & 280 \\
\hline Nigéria & 1991 & 139 & 1.041 & ** & 270 \\
\hline Nepal & 2000 & 46 & 1.187 & ** & 240 \\
\hline Vietnã & 1989 & 54 & 0.976 & ** & 220 \\
\hline Tanzânia & 1988 & 32 & 1.010 & & 190 \\
\hline Moçambique & 1997 & 33 & 0.859 & ** & 180 \\
\hline Etiópia & 1994 & 63 & 1.065 & & 110 \\
\hline \multicolumn{3}{|c|}{ Média Países em Desenvolvimento } & 1.033 & & 2101 \\
\hline \multicolumn{3}{|l|}{ Desvio Padrão } & 0.160 & & 2120 \\
\hline
\end{tabular}

* Significante a $5 \%$ para $\beta \neq 1 e^{* *}$ Significante a $1 \%$ para $\beta \neq 1$

Fonte: Elaboração própria a partir de Soo (2005) e World Bank (2003). 
Existem ainda outras explicações para as diferenças detectadas acima. Alguns estudiosos observam que a existência de imensas cidades nos países menos desenvolvidos não seria uma fase do processo de desenvolvimento econômico, mas um resultado indireto e não-desejado de políticas de substituição de importações que restringem o acesso aos mercados externos e garante o poder de mercado a produtores domésticos e estimulam a polarização urbana. ${ }^{7}$

Outros comentadores sobre redes de cidades notam também que uma relativa estabilidade na distribuição de Pareto não significa estabilidades microurbanas. Na evolução dos sistemas urbanos existem duas dinâmicas: uma que atua no nível macro, e que caracteriza a evolução da distribuição do tamanho das cidades, e uma segunda, no nível micro, que atinge as cidades individualmente. Esses analistas argumentam que existem vários exemplos históricos que combinam fortes instabilidades microurbanas com estabilidades macrourbanas. 8

Por exemplo, nos últimos dois séculos os EUA passaram por profundas mudanças na sua estrutura urbana. Los Angeles (CA), Dallas (TX) e Seattle (WA) eram aglomerados urbanos menores no final do século XIX e se transformaram em grandes centros urbanos em meados do século XX. O oposto ocorreu com New Haven (CT), New Bradford (MA) e Dumphries (VA). Mais recentemente, nas décadas dos 60 e 70, novamente várias cidades americanas perderam imensos contingentes populacionais, tais como Detroit (MI), Philadelphia (PA), Baltimore (MD) e Chicago (IL), enquanto Phoenix (AZ), Houston (TX) e San Jose (CA), entre outras, cresceram aceleradamente. Apesar destas transformações, não se verificaram mudanças significativas no coeficiente de Pareto para os EUA.

Alguns modelos que lidam explicitamente com a organização da produção no espaço, como os de Allen (1997), Fujita et al. (1999) e Henderson (1974 e 1988), são capazes de gerar estruturas urbanas que se aproximam de estruturas Zipf. Contudo, esses modelos demandam parametrizações específicas, para a qual não existem argumentos teóricos precisos e explícitos. Como observa Fujita, Krugman e Venables (1999, p. 225), este é ainda um desafio para os estudiosos da área de economia regional e urbana.

Não obstante as polêmicas em torno do exato valor dos coeficientes de Pareto, a maioria dos autores considera o expoente de Pareto ( $\beta$ ) uma ótima descrição das estruturas urbanas e que permite a comparação, até mesmo temporal, de diversos países e estruturas regionais.

7 Ver Alonso-Villar (2001), Krugman (1996), Krugman e Livas (1996) e Puga (1998).

8 Ver Storper e Walker (1989) e Guérin-Pace (1995). 
Além do coeficiente de Pareto, outra medida tradicional de concentração e assimetria urbana é a primazia das cidades. A primazia das cidades é basicamente uma medida de tamanho relativo: a porcentagem da população da principal cidade em relação à soma das populaçôes das 5, 10 ou mesmo 50 maiores cidades da amostra:

$$
\mathrm{P}_{5}=\mathrm{N}_{1} /\left(\mathrm{N}_{1}+\ldots+\mathrm{N}_{5}\right)
$$

$\mathrm{N}_{\mathrm{i}}$ : população da cidade $\mathrm{i}$

É com base nesses dois convencionais índices de concentração e assimetria (primazia e coeficiente de Pareto) que este trabalho avaliará as estruturas urbanas do Brasil e Estados Unidos. ${ }^{9}$

\section{AS ESTRUTURAS URBANAS DO BRASIL E ESTADOS UNIDOS}

Várias são as diferenças e similaridades entre Brasil e Estados Unidos da América. Entre as similaridades podem ser destacadas: (1) são países continentais, (2) e constituídos por "populações transplantadas" (imigrantes e escravos), (3) têm populações acima de 150 milhões de habitantes (4) e apresentam elevados graus de urbanização, o que garante uma ampla rede de cidades. Dentre as diferenças podem ser citadas: (1) a distribuição da renda, (2) o nível de renda e o tamanho do mercado interno, (3) a capacidade tecnológica, (4) o padrão de internacionalização da economia, (5) a estrutura financeira. Por estas e outras tantas características, esses dois países podem apresentar simetrias e assimetrias na estrutura urbana.

Para dar início à análise, vale notar que $90 \%$ da população urbana dos EUA está localizada em apenas 717 cidades; já no caso do Brasil, essa mesma população está dispersa em 1.555 diferentes cidades ou regiões metropolitanas. Esta dispersão populacional brasileira não corresponde a uma menor polarização urbana, como será ilustrado. ${ }^{10}$

No caso do Brasil, os núcleos urbanos serão definidos como cidades e regiôes metropolitanas (RM); para os EUA, as consolidated metropolitan statistical area (CMSA) serão a referência para as grandes aglomeraçôes. A Tabela 3 apresenta medidas de primazia para os dois países e a Figura 1 mostra as distribuiçóes de Pareto (log-lineares) do tamanho da população e ranking das cidades. Como se pode notar, o Brasil apresenta

9 Outro índice de desconcentração urbana muito utilizado é o de Wheaton e Shishido (1979): o inverso do clássico índice de concentração de Herfindahl e Hirschman. Este índice não será utilizado, pois em vários casos foram gerados valores não conclusivos e/ou similares às primazias e coeficientes de Pareto.

10 A base de dados para essa comparação são a população dos municípios brasileiros publicadas no Censo Demográfico do Brasil 2000 / IBGE, e no caso dos EUA, a população dos counties de 1998 publicadas pelo BEA - Burean of Economics Analysis. 
medidas de primazia superiores às dos EUA e um viés em favor de uma maior polarização urbana.

A Tabela 4 apresenta estimativas dos coeficientes de Pareto ( $\beta$ ) para EUA e Brasil para diferentes cortes nas populações urbanas. No caso das 100 maiores aglomeraçóes urbanas, o Brasil apresenta um coeficiente de $\beta=0.89$, enquanto os EUA têm um coeficiente $\beta=1.04$. Poder-se-ia dizer, assim, que as medidas de concentração capturadas pelos coeficientes de Pareto e primazias indicam o Brasil como um país mais polarizado que os EUA.

Outro aspecto interessante da Tabela 4 são as mudanças no valor de $\beta$ para diferentes cortes na população urbana. No caso dos EUA, quando as cidades menores são progressivamente excluídas da amostra, o coeficiente de Pareto aumenta de $\beta=0.68$ ( $100 \%$ da população urbana) para $\beta=1.22$ (50\% da população urbana). Há, portanto, um progressivo processo de desconcentração na parte superior do ranking urbano norte-americano e a presença de um número maior de cidades médias na estrutura urbana dos EUA.

\section{FIGURA 1 - DISTRIBUIÇÕES DE CIDADES - BRASIL E EUA}

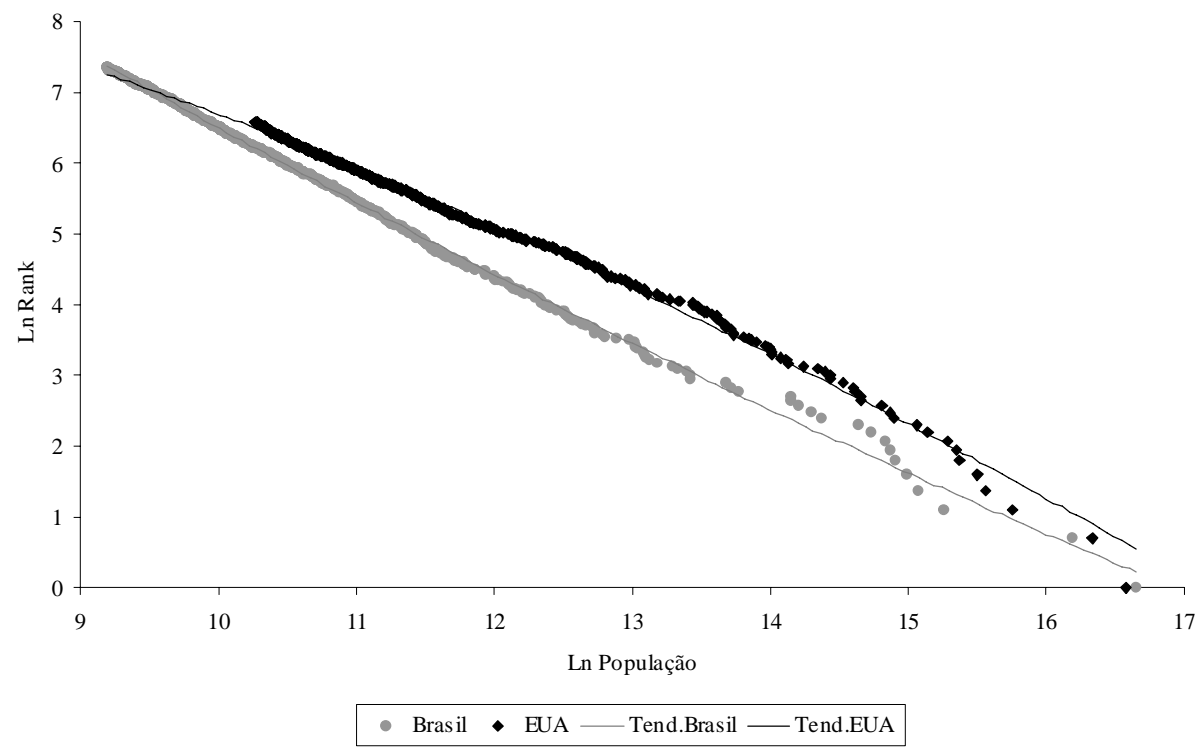

Nota: Distribuição com $90 \%$ da população urbana.

Fonte: Elaboração própria a partir de IBGE (2000) e BEA (2000). 
TABELA 3 - BRASIL E EUA - PRIMAZIAS URBANAS

\begin{tabular}{lll}
\hline Primazias & Brasil & EUA \\
\hline Primazia 5 & 0.440 & 0.341 \\
Primazia 10 & 0.327 & 0.235 \\
Primazia 30 & 0.247 & 0.151 \\
\hline
\end{tabular}

Fonte: Elaboração própria a partir de IBGE (2000) e BEA (2000).

TABELA 4 - BRASIL E EUA - COEFICIENTES DE PARETO $(\beta)$

\begin{tabular}{lcccccc}
\hline & \multicolumn{3}{c}{ Brasil } & & \multicolumn{2}{c}{ EUA } \\
\cline { 2 - 3 } \cline { 5 - 6 } População Urbana & $\beta$ & $\mathrm{R}^{2}$ & & $\beta$ & $\mathrm{R}^{2}$ \\
\hline $100 \%$ & 0.737 & 0.920 & & 0.681 & 0.914 \\
$95 \%$ & 1.005 & 0.997 & & 0.877 & 0.995 \\
$90 \%$ & 1.028 & 0.997 & & 0.860 & 0.993 \\
$80 \%$ & 1.002 & 0.994 & & 0.892 & 0.984 \\
$70 \%$ & 0.946 & 0.992 & & 1.021 & 0.985 \\
$60 \%$ & 0.884 & 0.987 & & 1.156 & 0.987 \\
$50 \%$ & 0.868 & 0.963 & & 1.222 & 0.981 \\
100 maiores & 0.889 & 0.989 & & 1.039 & 0.983 \\
aglomerações & & & & &
\end{tabular}

Fonte: Elaboração própria a partir de IBGE (2000) e BEA (2000).

No caso brasileiro observa-se uma situação peculiar: não há uma redução progressiva do coeficiente de Pareto, mas sim um aumento. Para 100\% da população urbana o coeficiente de Pareto é $\beta=0.74$ e para $90 \%$ da população $\beta=1.03$, o que sinaliza uma certa desconcentração urbana. Contudo, quando menos de $90 \%$ da população é incluída na amostra, o coeficiente de Pareto passa a indicar um processo de concentração urbana. Ao final, com $50 \%$ da população o coeficiente de Pareto é $\beta=0.87$. Este decréscimo de $\beta$ indica uma concentração populacional no extrato superior do ranking das cidades e uma certa fragilidade das cidades médias na estrutura urbana do Brasil.

Essa análise das estruturas urbanas mostra que o Brasil apresenta uma polarização urbana superior à dos EUA. Para detalhar um pouco mais as diferenças, introduziu-se uma terceira variável não-linear nas estimativas do coeficiente de Pareto:

$$
\operatorname{Ln} R_{i}=(\operatorname{Ln} \alpha)-\beta_{1}\left(\operatorname{Ln} N_{i}\right)+\beta_{2}\left(\operatorname{Ln} N_{i}\right)^{2}
$$


Essa relação não-linear foi estimada para três grupos populacionais: o primeiro corresponde a $90 \%$ da população urbana e o segundo e terceiros seriam cidades com mais de 20 e 50 mil habitantes. Existem duas razões para explicar essas diferentes segmentaçôes. O primeiro corte visa excluir as pequenas áreas urbanas da amostra, pois a lei de Zipf é válida somente para os extratos superiores da distribuição de cidades; a "cauda" da distribuição não segue esta regra. Poder-se-ia utilizar até mesmo $50 \%$ da população, mas optou-se por $90 \%$, pois assim são destacadas com mais detalhes as diferenças entre as redes de cidades nos dois países.

O segundo corte tem como referência um debate contemporâneo sobre as características das áreas urbanas e rurais. Veiga $(2005,2004 a)$ registra e comenta esta polêmica, afirmando que as estatísticas oficiais - particularmente a brasileira - tendem a superestimar a população urbana brasileira. Argumenta que a classificação oficial não leva em consideração a estrutura funcional da aglomeração, a existência de amenidades e infraestruturas que a qualificassem como uma área urbanizada. Pelo lado ambiental, essa classificação também não incorpora as alterações provocadas pela aglomeração humana no ecossistema, um critério certamente relevante para definir os impactos antrópicos; uma medida para esse impacto seria a densidade demográfica (hab. $/ \mathrm{km}^{2}$ ).

No que tange à clássica tipologia urbano e rural, o autor destaca que as atuais áreas rurais não correspondem às antigas regiões agrárias. As "novas áreas rurais" não são predominantemente agrícolas, são economicamente dinâmicas, possuem uma infraestrutura habitacional e acessibilidade que as mantêm fortemente conectadas aos centros urbanos. Essas regiões não seriam certamente urbanas, no sentido tradicional da palavra, mas também não se encaixariam na antiga definição do rural-agrário. ${ }^{11}$

Tendo como referência esse debate, optou-se por um segundo corte, tanto para o Brasil como para os EUA: foram excluídas as cidades com menos de 20 e 50 mil habitantes, um critério que objetiva excluir as "cidades oficiais", as "cidades não-urbanas" e as "cidades semi-urbanas". Tomando-se o corte 20 mil habitantes, no Brasil existiriam 741 núcleos urbanos (66\% da população), enquanto que os EUA teriam 1.013 áreas urbanas (74\% da população). Quando o corte é 50 mil habitantes, o

11 Para uma sumária apreciação do debate sobre as definições, conexões e contradiçôes do urbano e do rural, ver Veiga (2004b e 2004c) e Monte-Mor (2001 e 2005). À guisa de ilustração sobre as controvérsias neste debate, Monte-Mor (2005, p. 947) argumenta que, sob certo ponto vista, "o Brasil é todo urbano": "In this broader sense, one can speak of an extensive urbanization imposing itself on Brazilian space far beyond the cities, integrating rural and regional spaces with urban-industrial space through the expansion of the material base required by the contemporary society and economy and the relations of production which are (or should be) reproduced by the production of space itself." 
Brasil passaria a ter apenas 290 áreas urbanas (58\% da população) e os EUA 420 (67\% da população). ${ }^{12}$

Como se observa nas Tabelas 5 e 6, as relações não-lineares entre os tamanhos das cidades e suas posições no ranking são significativas. A única exceção é o corte 20 mil habitantes para o Brasil, onde a relação é linear. Mais interessante ainda são as estruturas urbanas descritas pelas regressões. As regressóes da Tabela 5 apresentam a estrutura brasileira como convexa, enquanto que no caso americano a estrutura é côncava ( $\beta_{2}$ com sinais opostos). Quanto o corte é 50 mil habitantes, as regressões apresentam os coeficientes $\beta_{1}$ e $\beta_{2}$ com sinais opostos. Novamente, têm-se indicações das diferenças já anunciadas na análise anterior: a maior polarização na rede de cidades brasileira vis-àvis à norte-americana.

TABELA 5 - BRASIL - RELAÇ $\tilde{A} O$ N $\tilde{A O}-L I N E A R$

\begin{tabular}{|c|c|c|c|}
\hline \multicolumn{4}{|c|}{ (90 \% da População Urbana) } \\
\hline & Coeficiente & Desvio Padrão & Valor de T \\
\hline$\overline{\operatorname{Ln} \alpha}$ & $19.20^{*}$ & 0.06 & 322.18 \\
\hline$\beta_{1}$ & $-1.46^{*}$ & 0.01 & -136.30 \\
\hline$\beta_{2}$ & $0.02^{*}$ & 0.01 & 40.83 \\
\hline \multicolumn{4}{|c|}{$\mathrm{R}^{2}=0.998,1.555$ localidades (73\% da população) } \\
\hline \multicolumn{4}{|c|}{ Cidades com mais de 20 mil habitantes } \\
\hline & Coeficiente & Desvio Padrão & Valor de T \\
\hline $\operatorname{Ln} \alpha$ & 12.67 & 17857.78 & 0.00 \\
\hline$\beta_{1}$ & $-1.00^{*}$ & 0.00 & -406.16 \\
\hline$\beta_{2}$ & 51.08 & 235490.6 & 0.00 \\
\hline \multicolumn{4}{|c|}{$\mathrm{R}^{2}=0.995,741$ localidades (66\% da população) } \\
\hline \multicolumn{4}{|c|}{ Cidades com mais de 50 mil habitantes } \\
\hline & Coeficiente & Desvio Padrão & Valor de T \\
\hline $\operatorname{Ln} \alpha$ & $21.57^{\star}$ & 0.40 & 53.54 \\
\hline$\beta_{1}$ & $-1.85^{\star}$ & 0.06 & -29.01 \\
\hline$\beta_{2}$ & $0.03^{\star}$ & 0.01 & 13.94 \\
\hline
\end{tabular}

* significativo a $1 \%$.

Fonte: Elaboração própria a partir de IBGE (2000).

12 É certo que segmentar o urbano e o não-urbano a partir da população da localidade não é único e nem o melhor critério, ainda mais quando se comparam dois países tão distintos como EUA e Brasil. Logo, devese considerar esse critério como uma primeira aproximação para efeitos comparativos. 
TABELA 6 - EUA - RELAÇÃO NÃO-LINEAR

\begin{tabular}{|c|c|c|c|}
\hline \multicolumn{4}{|c|}{ (90 \% da População Urbana) } \\
\hline & Coeficiente & Desvio Padrão & Valor de T \\
\hline $\operatorname{Ln} \alpha$ & $11.10^{*}$ & 0.19 & 55.74 \\
\hline$\beta_{1}$ & $-0.15^{\star}$ & 0.03 & -4.30 \\
\hline$\beta_{2}$ & $-0.03^{*}$ & 0.01 & -22.49 \\
\hline \multicolumn{4}{|c|}{$\mathrm{R}^{2}=0.996,717$ localidades ( $71 \%$ da população) } \\
\hline \multicolumn{4}{|c|}{ Cidades com mais de 20 mil habitantes } \\
\hline & Coeficiente & Desvio Padrão & Valor de T \\
\hline $\operatorname{Ln} \alpha$ & $13.90^{*}$ & 0.17 & 83.04 \\
\hline$\beta_{1}$ & $-0.59^{\star}$ & 0.03 & -21.07 \\
\hline$\beta_{2}$ & $-0.01^{*}$ & 0.01 & -9.96 \\
\hline \multicolumn{4}{|c|}{$\mathrm{R}^{2}=0.995,1.013$ localidades ( $74 \%$ da população) } \\
\hline \multicolumn{4}{|c|}{ Cidades com mais de 50 mil habitantes } \\
\hline & Coeficiente & Desvio Padrão & Valor de T \\
\hline $\operatorname{Ln} \alpha$ & $6.91^{*}$ & 0.27 & 25.31 \\
\hline$\beta_{1}$ & $0.49^{*}$ & 0.04 & 11.37 \\
\hline$\beta_{2}$ & $-0.05^{*}$ & 0.01 & -31.60 \\
\hline
\end{tabular}

* significativo a $1 \%$.

Fonte: Elaboração própria a partir de BEA (2000).

Para melhor ilustrar as diferenças detectadas acima, uma simulação foi elaborada. Dado que a população do Brasil é de aproximadamente 170 milhões de indivíduos e a dos EUA alcança os 270 milhões de habitantes, temos um efeito escala que tende a dificultar a visualização das diferenças entre os países. Assim, ajustando a população dos EUA à população brasileira e estimando a nova estrutura urbana desse "EUA fictício" obtemos as seguintes regressões para os dois países: 
TABELA 7 - SIMULANDO EUA COM ESCALA BRASIL

\begin{tabular}{|c|c|c|c|c|}
\hline País & Constante & Ln Pop & $(\operatorname{Ln} P o p)^{2}$ & $\mathrm{R}^{2}$ \\
\hline \multicolumn{5}{|c|}{ 90\% da População Urbana } \\
\hline Brasil & +19.20 & -1.46 & +0.02 & 0.998 \\
\hline EUA & +11.03 & -0.18 & -0.03 & 0.996 \\
\hline \multicolumn{5}{|c|}{ Cidades com mais de 20 mil habitantes ${ }^{*}$} \\
\hline Brasil & +16.54 & -1.00 & --- & 0.995 \\
\hline EUA & +13.57 & -0.06 & -0.01 & 0.995 \\
\hline \multicolumn{5}{|c|}{ Cidades com mais de 50 mil habitantes } \\
\hline Brasil & +21.57 & -1.85 & +0.03 & 0.995 \\
\hline EUA & +7.19 & +0.42 & -0.05 & 0.997 \\
\hline
\end{tabular}

* No caso brasileiro a relação é linear. Ver Tabela 5.

Fonte: Elaboração Própria a partir de BEA (2000) e IBGE (2000).

A principal diferença entre estas equações está no sinal do coeficiente do termo não-linear $(\operatorname{Ln} \mathrm{N})^{2}$. No caso EUA, o sinal é negativo, logo, este termo tende a intensificar a relação inversa entre o tamanho e ranking da população e assim gerar uma distribuição côncava. No caso brasileiro, o sinal do coeficiente de $(\mathrm{Ln} \mathrm{N})^{2}$ é positivo e atua em favor de uma distribuição convexa. Para o extrato superior da hierarquia urbana (cidades com mais de 50 mil habitantes), as diferenças são as mais acentuadas e podem ser vistas na Figura 2. No caso dos EUA, as cidades médias são maiores que as brasileiras e as cidades pequenas tendem a ser menores. Logo, estruturas urbanas similares à norteamericana são menos polarizadas que outras similares à brasileira, em que há uma predominância dos maiores centros urbanos sobre as cidades médias e pequenas.

A fragilidade das cidades médias produz distorções nas distribuições de cidades e rompem uma esperada relação log-linear entre tamanho e ranking das cidades. Esta característica é ainda mais aparente nos estados e regiões brasileiras. De modo geral, todas as estruturas urbanas estaduais e regionais apresentam "desequilíbrios" similares que refletem uma polarização acentuada combinada com uma ausência de cidades médias. Esta destacada fragilidade das cidades médias brasileiras já foi observada em outros estudos. Por exemplo, Andrade e Serra (1998 e 1999) e Pereira e Lemos (2003) anunciam, de forma variada, a ainda delicada posição das cidades médias nas estruturas urbanas brasileiras. 
FIGURA 2 - SIMULANDO EUA COM ESCALA BRASIL*

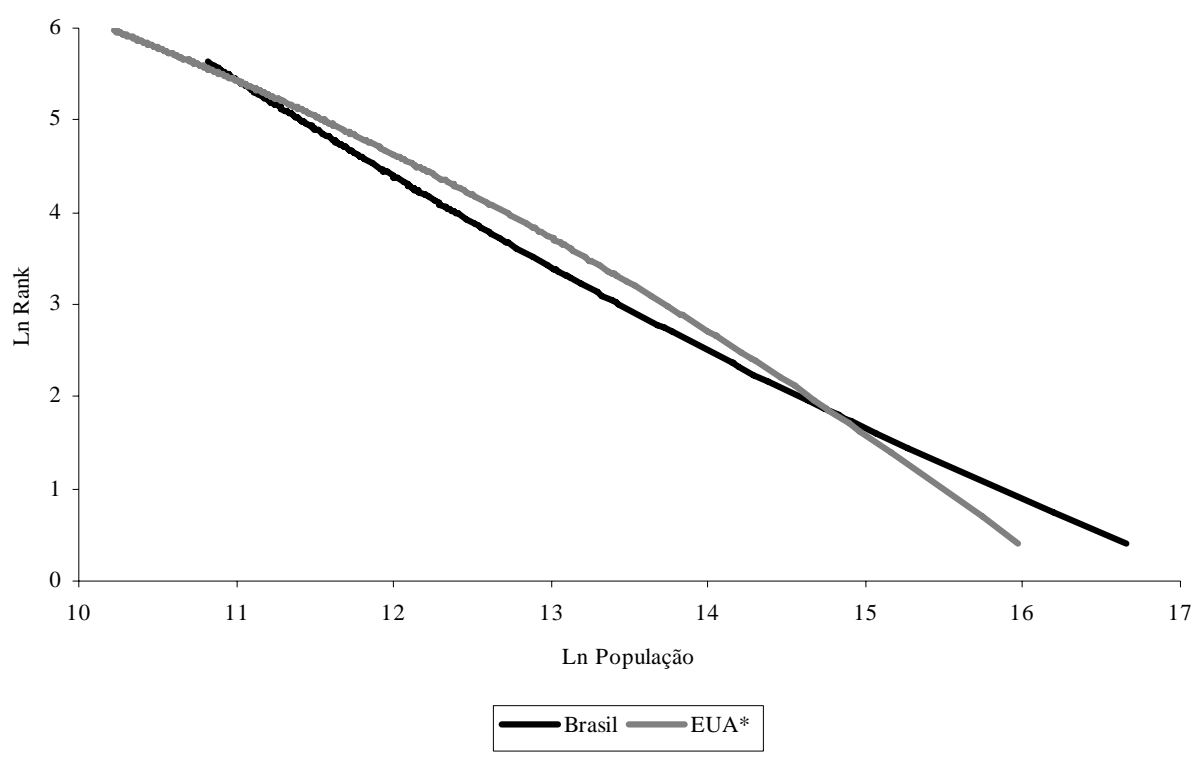

* Cidades com mais de 50 mil habitantes.

Fonte: Elaboração Própria a partir de IBGE (2000) e BEA (2000).

\section{DIVERGÊNCIAS EM RELAÇÃO ÀS HIERARQUIAS URBANAS ZIPF}

Como comentado na parte inicial desse artigo, para se gerar uma distribuição Zipf em economia urbana uma hipótese é fundamental: todas as áreas urbanas devem possuir as mesmas potencialidades, pouco importando o seu tamanho ou posição no espaço econômico. Assim, caso as cidades tenham as mesmas chances de crescer, uma distribuição Zipf emergiria ao final de um certo período de tempo. Entretanto, se algumas cidades forem mais ou menos favorecidas, a distribuição de tamanho se afastará da distribuição Zipf $(\beta \neq 1)$. Portanto, divergências em relação a $\beta=1$ podem indicar "oportunidades de crescimento diferenciadas” entre os núcleos urbanos.

Gabaix (1999) apresenta um modelo em que, no momento inicial, as cidades possuem os mesmos tamanhos e taxas de crescimento populacional. Quando essas taxas são associadas a choques exógenos e estocásticos com as mesmas características (mesmo desvio padrão), a emergência de uma distribuição Zipf é certa e ocorrerá em poucos períodos. Contudo, quando existem oportunidades de crescimento diferenciadas e favoráveis às grandes cidades, o coeficiente de Pareto será $\beta<1$, mas quando as oportunidades são favoráveis às pequenas e médias cidades, $\beta>1$. Em outras palavras: as economias e deseconomias urbanas não se equivalem e distorcem as taxas de crescimento das cidades e a estrutura urbana. 
Até o momento, a principal conclusão empírica deste trabalho é que as estruturas urbanas do Brasil e EUA são diferenciadas: a brasileira é mais concentrada (convexa) e a norte-americana é menos polarizada (côncava). Há uma fragilidade na estrutura urbana brasileira: as cidades médias são pequenas - ou existem em pequeno número - e a polarização urbana é mais acentuada em favor das maiores cidades. No caso dos EUA, o oposto ocorre: há uma marcante presença de cidades médias na estrutura urbana.

Combinando essa conclusão empírica com os argumentos teóricos extraídos da leitura de Gabaix (1999), pode-se afirmar que as cidades médias não possuem as mesmas oportunidades de crescimento que as grandes cidades brasileiras. No caso brasileiro, até o período recente, as economias de aglomeração suplantaram as deseconomias de aglomeração e favoreceram o surgimento de grandes cidades, enquanto as pequenas não se desenvolveram ou estão subdimensionadas. Nos EUA, o inverso se verifica: as oportunidades de crescimento tendem a favorecer as cidades médias.

Para quantificar o impacto dessas diferentes oportunidades de crescimento na estrutura urbana foram simuladas estruturas urbanas "ideais" para o Brasil e os EUA. Para tanto, foram adotados os seguintes pressupostos: primeiro, manteve-se o ranking das cidades como apresentado no Censo Demográfico de 2000 e BEA (2000); segundo, mantevese fixo o número de cidades com mais de 50 mil habitantes (290 cidades e população de aproximadamente 99 milhóes de habitantes) e dos EUA (420 cidades e aproximadamente 181 milhões de habitantes). A partir desses dois pressupostos, ajustou-se a hierarquia de cidades à distribuição de Zipf $(\beta=1)$, obtendo-se, assim, as projeções do tamanho ideal de cidades para os dois países.

A Figura 3 mostra a distribuição Zipf para o Brasil e a Tabela 8 apresenta as quarenta maiores cidades (ou regiões metropolitanas) e o seu correspondente tamanho ideal. A Tabela 9 e Figura 4 têm as mesmas informações para o caso dos EUA. No caso brasileiro, a simulação mostra Belo Horizonte e Porto Alegre abaixo do seu tamanho potencial, enquanto São Paulo e Rio de Janeiro e outras regióes metropolitanas como superdimensionadas. Dentre essas outras cidades, vale citar Campinas, Curitiba e Distrito Federal, todas apresentando dimensões superiores à esperada em relação à distribuição de Zipf. Outro aspecto que merece destaque é o subdimensionamento de cidades localizadas abaixo de Manaus (posição 15). É exatamente a partir desta posição que as cidades médias mais fragilizadas surgem. A Figura 3 fornece uma visão geral da imensa massa de cidades médias que estão abaixo do seu tamanho potencial. 
FIGURA 3 - DISTRIBUIÇÃO DE CIDADES DO BRASIL E NA ZIPF BRASIL $2000^{*}$

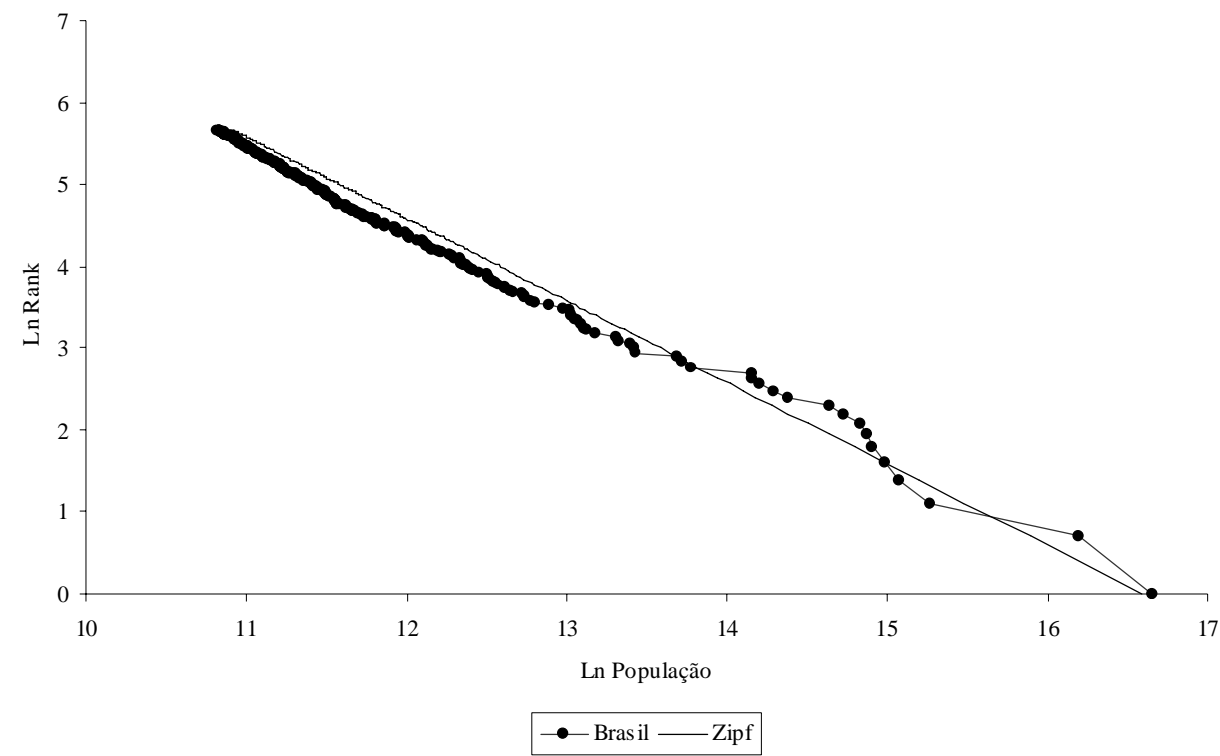

* Cidades com mais de 50 mil habitantes.

Fonte: Elaboração própria a partir de IBGE (2000).

FIGURA 4 - DISTRIBUIÇÃO DE CIDADES DOS EUA E NA ZIPF EUA - 1998*

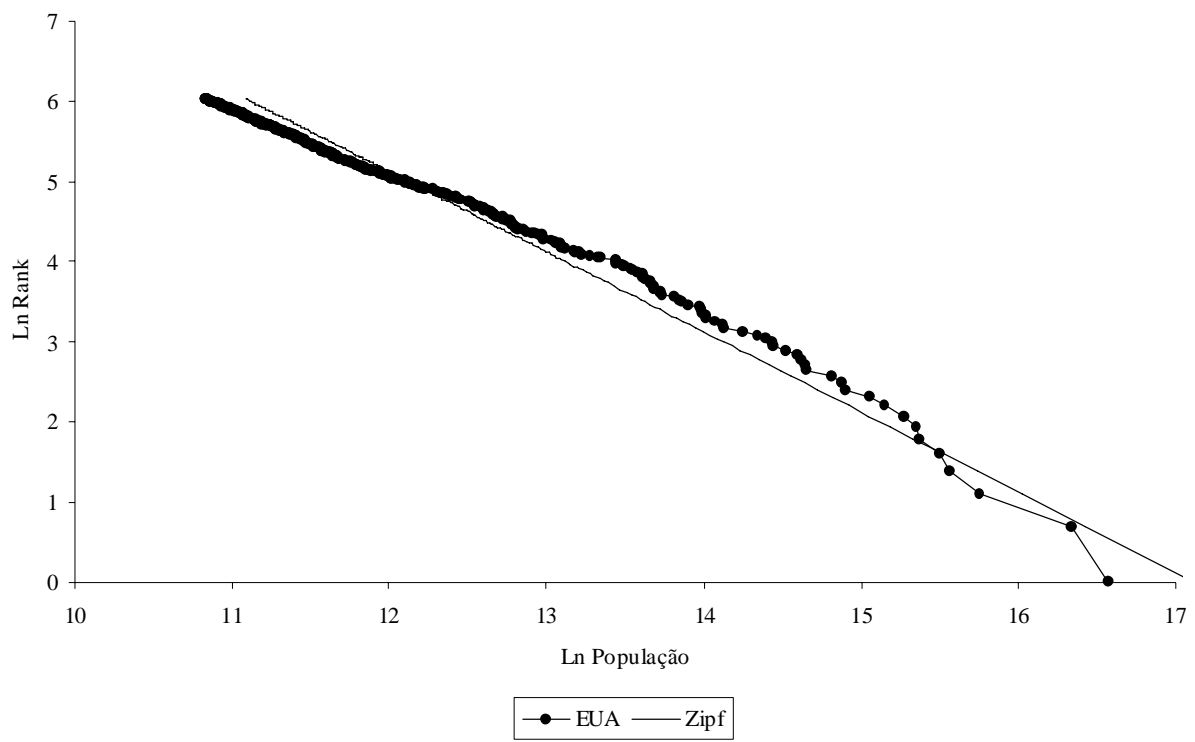

* Cidades com mais de 50 mil habitantes.

Fonte: Elaboração própria a partir de BEA (2000). 
No caso dos EUA, as deseconomias de aglomeração já estão presentes no extrato superior da rede de cidades. $\mathrm{O}$ aparente subdimensionamento das suas quatro maiores regióes metropolitanas (New York, Los Angeles, Chicago e Washington) ilustra essa limitação à expansão da escala urbana. A região metropolitana de Nova York pode estar subdimensionada nas estatísticas oficiais, e alguns estudos já estimam a população dessa mancha urbana em mais de 20 milhões de habitantes. Mesmo que essas estimativas fossem mais adequadas, a escala de Nova York ainda estaria abaixo do "ideal". Portanto, pode-se afirmar que os EUA têm uma estrutura urbana oposta à brasileira: suas cidades médias apresentam escalas superiores ao esperado. Na simulação, todas as cidades abaixo de Washington (posição 4) estão superdimensionadas (Tabela 9).

Os "super" e "sub" dimensionamentos podem indicar que algumas cidades estão crescendo (ou cresceram) mais rapidamente que outras. Assim, a hierarquia urbana estaria em mutação: algumas cidades ditas superdimensionadas estariam ascendendo ao extrato superior do ranking e deslocando outras para o extrato inferior. No caso de Belo Horizonte e de Porto Alegre, seus subdimensionamentos indicariam um processo de ascensão ainda inconcluso ou mesmo o seu inverso: uma estagnação e a emergência de outras cidades. Qualquer um desses dois movimentos geraria subdimensionamentos em relação à hierarquia capturada do Censo Demográfico de 2000.

A interpretação acima ajudaria na explicação de mudanças microurbanas pontuais na estrutura brasileira e na compreensão da dinâmica de rede de cidades regionais. Contudo, o argumento deste trabalho - e que é compartilhado por vários analistas - é que existem fragilidades estruturais na rede de cidades brasileira: as cidades de pequeno e médio portes não apresentaram, até o momento, as mesmas oportunidades de crescimento presentes ou ofertadas às grandes cidades e regiôes metropolitanas.

Como dito, essas afirmações se baseiam em simulações com pressupostos muito rígidos, tais como a estabilidade do ranking e da hierarquia de cidades. Contudo, para o caso brasileiro, este estudo sinaliza um ponto fundamental: para se manter no extrato superior da hierarquia urbana nacional, cidades como Belo Horizonte e Porto Alegre têm que crescer em termos populacionais. A escala dessas cidades não lhes garante uma posição sólida no cenário urbano brasileiro, o mesmo argumento valendo para outras cidades médias subdimensionadas e listadas na Tabela 8. Quanto às regiốes metropolitanas acima do tamanho ideal, o inverso se verificou: estas foram favorecidas, seja por meio de uma política de desenvolvimento pouco voltada para a descentralização ou mesmo pelo padrão de industrialização fordista que caracterizou a urbanização brasileira. ${ }^{13}$

13 As vantagens das grandes cidades podem ser resultado de diversos determinantes. Existe uma longa lista de fatores, que se estende desde oferta de infra-estrutura até modelos de desenvolvimento com pouca ênfase na desconcentração. Ver Alperovich (1993) e Soo (2005) para estudos empíricos sobre possíveis determinantes da estrutura urbana. 
TABELA 8 - AS 40 MAIORES CIDADES NA DISTRIBUIÇÃO ZIPF BRASIL 2000

\begin{tabular}{|c|c|c|c|}
\hline Região Metropolitana ou Cidade & $\begin{array}{l}\text { Censo } 2000 \\
\text { (a) }\end{array}$ & $\begin{array}{l}\text { Zipf } \\
\text { (b) }\end{array}$ & $\begin{array}{c}\text { Diferença } \\
(a-b)\end{array}$ \\
\hline 1 - RM São Paulo & 17119400 & 15880608 & 1238792 \\
\hline 2 - RM Rio de Janeiro & 10813717 & 7940304 & 2873413 \\
\hline 3 - RM Belo Horizonte & 4242910 & 5293536 & -1050626 \\
\hline 4 - RM Porto Alegre & 3509384 & 3970152 & - 460768 \\
\hline 5 - RM Recife & 3234647 & 3176122 & 58525 \\
\hline 6 - RM Salvador & 2973880 & 2646768 & 327112 \\
\hline 7 - RM Fortaleza & 2881264 & 2268658 & 612606 \\
\hline 8 - RM Distrito Federal & 2755270 & 1985076 & 770194 \\
\hline 9 - RM Curitiba & 2500105 & 1764512 & 735593 \\
\hline 10 - RM Campinas & 2269718 & 1588061 & 681657 \\
\hline 11 - RM Belém & 1754786 & 1443692 & 311094 \\
\hline 12 - RM Goiânia & 1612874 & 1323384 & 289490 \\
\hline 13 - RM Baixada Santista & 1470774 & 1221585 & 249189 \\
\hline 14 - RM Vitória & 1401716 & 1134329 & 267387 \\
\hline 15 - Manaus & 1396768 & 1058707 & 338061 \\
\hline 16 - RM Maceió & 955173 & 992538 & -37365 \\
\hline 17 - RM Natal & 911552 & 934153 & -22601 \\
\hline 18 - RM São Luís & 877387 & 882256 & -4869 \\
\hline 19 - Teresina & 677470 & 835821 & -158351 \\
\hline 22 - RM Florianópolis & 673185 & 794030 & -120845 \\
\hline 21 - Campo Grande & 655914 & 756219 & -100305 \\
\hline 22 - RM Londrina & 614138 & 721846 & -107708 \\
\hline 23 - João Pessoa & 597934 & 690461 & - 92527 \\
\hline 24 - São José dos Campos & 532717 & 661692 & - 128975 \\
\hline 25 - Ribeirão Preto & 502760 & 635224 & - 132464 \\
\hline 26 - Uberlândia & 488982 & 610793 & - 121811 \\
\hline 27 - Sorocaba & 486726 & 588171 & -101445 \\
\hline 28 - Cuiabá & 476532 & 567165 & -90633 \\
\hline 29 - Aracaju & 461534 & 547607 & -86073 \\
\hline 30 - Juiz de For a & 453002 & 529354 & -76352 \\
\hline 31 - RM Maringá & 452564 & 512278 & -59714 \\
\hline 32 - RM Norte/Nordeste Catarinense & 449596 & 496269 & -46673 \\
\hline 33 - Feira de Santana & 431730 & 481231 & - 49501 \\
\hline 34 - RM Vale do Aço & 395657 & 467077 & -71420 \\
\hline 35 - Campos dos Goytacazes & 364177 & 453732 & -89555 \\
\hline 36 - RM Vale do Itajaí & 355422 & 441128 & -85706 \\
\hline 37 - Campina Grande & 337484 & 429206 & -91722 \\
\hline 38 - São José do Rio Preto & 337289 & 417911 & -80622 \\
\hline 39 - Caxias do Sul & 333391 & 407195 & -73804 \\
\hline 40 - Piracicaba & 317374 & 397015 & -79641 \\
\hline
\end{tabular}

Fonte: Elaboração própria a partir de IBGE (2000). 
TABELA 9 - AS 40 MAIORES CIDADES NA DISTRIBUIÇÃO ZIPF EUA - 1998

\begin{tabular}{|c|c|c|c|}
\hline $\begin{array}{l}\text { Metropolitan Statistical Area or } \\
\text { Consolidated Statistical Areas }\end{array}$ & $\begin{array}{l}\text { BEA } 2000 \\
\text { (a) }\end{array}$ & $\begin{array}{l}\text { Zipf } \\
\text { (b) }\end{array}$ & $\begin{array}{c}\text { Diferença } \\
(a-b)\end{array}$ \\
\hline 1 - New York-New Jersey-Long Island & 15797820 & 27364751 & -11566931 \\
\hline 2 - Los Angeles-Riverside-Orange County & 12479162 & 13682376 & -1203214 \\
\hline 3 - Chicago-Gary-Kenosha & 6970219 & 9121584 & -2151365 \\
\hline 4 - Washington-Baltimore & 5743107 & 6841188 & -1098081 \\
\hline 5 - San Francisco-Oakland-San Jose & 5382543 & 5472950 & -90407 \\
\hline 6 - Philadelphia-Wilmington-Atlantic City & 4729454 & 4560792 & 168662 \\
\hline 7 - Boston-Worcester-Lawrence-Lowell-Brocktn & 4632291 & 3909250 & 723041 \\
\hline 8 - Detroit-Ann Arbor-Flint & 4307812 & 3420594 & 887218 \\
\hline 9 - Dallas-Fort Worth & 3787644 & 3040528 & 747116 \\
\hline 10 - Houston-Galveston-Brazoria & 3476544 & 2736475 & 740069 \\
\hline 11 - Atlanta & 2957777 & 2487705 & 470073 \\
\hline 12 - Miami-Fort Lauderdale & 2890331 & 2280396 & 609935 \\
\hline 13 - Seattle-Tacoma-Bremerton & 2703950 & 2104981 & 598970 \\
\hline 14 - Phoenix-Mesa & 2315274 & 1954625 & 360648 \\
\hline 15 - Cleveland-Akron & 2300990 & 1824317 & 476674 \\
\hline 16 - Minneapolis-St. Paul & 2236043 & 1710297 & 525746 \\
\hline 17 - San Diego & 2185237 & 1609691 & 575546 \\
\hline 18 - St. Louis & 2023700 & 1520264 & 503436 \\
\hline 19 - Denver-Boulder-Greeley & 1866636 & 1440250 & 426386 \\
\hline 20 - Pittsburgh & 1852660 & 1368238 & 484422 \\
\hline 21 - Tampa-St. Petersburg-Clearwater & 1780980 & 1303083 & 477897 \\
\hline 22 -Portland-Salem & 1698753 & 1243852 & 454900 \\
\hline 23 - Cincinnati-Hamilton & 1539200 & 1189772 & 349429 \\
\hline 24 - Kansas City & 1372415 & 1140198 & 232217 \\
\hline 25 - Sacramento-Yolo & 1348949 & 1094590 & 254359 \\
\hline 26 - Milwaukee-Racine & 1299660 & 1052490 & 247169 \\
\hline 27 - Norfolk-Virginia Beach-Newport News & 1224859 & 1013509 & 211350 \\
\hline 28 - San Antonio & 1216689 & 977313 & 239377 \\
\hline 29 - Indianapolis & 1199874 & 943612 & 256262 \\
\hline 30 - Orlando & 1187241 & 912158 & 275083 \\
\hline 31 - Columbus & 1163721 & 882734 & 280987 \\
\hline 32 - Charlotte-Gastonia-Rock Hill & 1092213 & 855148 & 237064 \\
\hline 33 - Las Vegas & 1043245 & 829235 & 214010 \\
\hline 34 - New Orleans & 1032079 & 804846 & 227233 \\
\hline 35 - Salt Lake City-Ogden & 998015 & 781850 & 216165 \\
\hline 36 - Greensboro-Winston-Salem-High Point & 922444 & 760132 & 162312 \\
\hline 37 - Nashville & 912933 & 739588 & 173345 \\
\hline 38 - Buffalo-Niagara Falls & 909677 & 720125 & 189552 \\
\hline 39 - Hartford & 876174 & 701660 & 174514 \\
\hline 40 - Austin-San Marcos & 872643 & 684119 & 188525 \\
\hline
\end{tabular}

Fonte: Elaboração própria a partir de BEA (2000). 


\section{REFLEXÕES FINAIS}

A principal conclusão deste trabalho é que na distribuição de tamanho de cidades do Brasil há uma predominância dos grandes centros urbanos, enquanto nos EUA as cidades médias tendem a ter uma presença mais marcante. Este fato é captado pelas opostas concavidades das distribuições de tamanho das cidades dos dois países.

Esta conclusão empírica, associada a uma discussão teórica sobre as condições que garantem a emergência de uma distribuição Zipf, permite afirmar que, no Brasil, as cidades médias não tiveram oportunidades de crescimento equivalentes às ofertadas para as grandes cidades: houve uma preferência por alguns espaços urbanos, em geral as grandes capitais estaduais. Com exceção de Belo Horizonte e Porto Alegre, todas as grandes cidades brasileiras estão superdimensionadas, enquanto que as cidades médias se apresentam subdimensionadas.

Do ponto de vista das políticas urbanas, este estudo indica que investimentos nas regióes não-metropolitanas deveriam ser priorizados com o intuito de reduzir uma concentração excessiva nos poucos e esparsos grandes centros urbanos. Políticas públicas voltadas à criação de infra-estrutura de comunicação, transporte e energia, saneamento básico, políticas de habitação, qualificação da mão-de-obra e de difusão de inovações (parques tecnológicos, centros de pesquisa e universidades) contribuiriam para a redução do elevado "custo de transporte" que atua em favor das grandes cidades e que, provavelmente, estimula a instalação de empresas nas regiões metropolitanas, reduz o poder de compra das populaçôes de pequenas e médias cidades e incentiva a migração para os grandes núcleos urbanos já consolidados.

Para finalizar, vale observar que deslocar e mudar uma rede de cidades não é uma tarefa banal e nem rotineira, como atestam as polêmicas discussóes em torno dos casos de Brasília e Manaus. Estes casos ilustram, com nitidez, a magnitude das mudanças nas bases econômica e urbana necessárias para que tal fato ocorra. Resta saber quais seriam os agentes capazes de implementar tais intervenções na estrutura urbana brasileira.

\section{REFERENCIAS}

Allen, P. M. Cities and regions as a self-organizing system - Models of complexity. Netherlands, Amsterdam: Gordon and Breach Science Publishers, 1997.

Alonso-Villar, M. Large metropolises in the third world: an explanation. Urban Studies, v. 38, n. 8, p. 1359-1371, 2001.

Alperovich, G. The size distribution of cities: on the empirical validity of the ranksize rule. Journal of Urban Economics 16, p. 232-239, 1984. 
. An exploratory model of city-size distribution: evidence from cross-country data. Urban Studies 30, p. 1591-1601, 1993.

Andrade, T.; Serra, R. V. Crescimento econômico nas cidades médias brasileiras. Revista Nova Economia, v. 9, n. 1, 1999.

. O recente desempenho das cidades médias no crescimento populacional urbano brasileiro. Texto para Discussão n. 554. Brasília: IPEA, 1998.

BEA. Regional economic information system 1969-98 (RCN-0250). US Department of Commerce - Bureau of Economic Analysis / Regional Economic Measurement Division. USA: Washington, 2000.

Carrol, G. National city size distributions: what do we know after 67 years of research? Progress in Human Geography 6, p. 1-43, 1982.

Cuberes, D. The rise and decline of cities. Texto para Discussão - Universidade de Chicago, Setembro de 2004.

Fujita, M.; Krugman, P.; Venables, A. J. Spatial economy - Cities, regions and international trade. Cambridge, Massachusetts; London, England: The MIT Press, 1999.

Gabaix, X. Zipf's Law for cities: an explanation. Journal of Economics, p. 739-767, August 1999.

Gabaix, X.; Ionnides, Y. The evolution of city size distributions. MIT and Tuffs University, 2003. Mimeografado.

Guerin-Pace, F. Rank-size distribution and the process of urban growth. Urban Studies, v. 32, n. 3, p. 551-562, 1995.

Henderson, J. V. The sizes and types of cities. American Economic Review 64, p. 640656, 1974.

. Urban development - Theory, fact and illusion. New York, Oxford: Oxford University Press, 1988.

IBGE. Censo demográfico do Brasil 2000. Rio de Janeiro: IBGE, 2000.

Krakover, S. Testing the turning-point hypothesis in city-size distribution: the Israeli situation re-examined. Urban Studies, v. 35, n. 12, p. 2183-2196, 1998.

Krugman, P. R.; Livas, R. E. Trade policy and the third world metropolis. Journal of Development Economics, v. 49, n. 1, p. 137-150, 1996.

Krugman, P. R. Urban concentrations: the role of increasing returns and transport costs. International Regional Science Review, v. 19, n. 1 \& 2, p. 5-30, 1996.

Monte-Mor, R. L. New urban frontiers: contemporary tendencies in Brazil's urbanization. Texto para Discussão do CEDEPLAR, n. 165. Belo Horizonte: CEDEPLAR, Universidade Federal de Minas Gerais, 2001.

What is the urban in the contemporary world? Cadernos de Sauide Priblica, Rio de Janeiro: Fundação Oswaldo Cruz, v. 21, n. 3, p. 942-948. 2005.

Naude, W. A.; Krugell, W. F. Are South Africa's cities too small? Cities, v. 20, n. 3, p. 175-180, 2003. 
Parr, J. B. A note on the size distribution od cities over time. Journal of Urban Economics 18, p. 199-212, 1985.

Pereira, F. M.; Lemos, M. B. Cidades médias brasileiras: características e dinâmicas urbano industriais. Pesquisa e Planejamento Econômico, Rio de Janeiro: IPEA, v. 33, n. 1, p. 127-165, 2003.

Puga, D. Urbanization patterns: European versus less developed countries. Journal of Regional Science 38, p. 231-252, 1998.

Reed, W. The Pareto, Zipf, and other power laws. Economics Letters 74, p. 15-19, 2001.

Richardson, H. W. Theory of the distribution of city sizes: review and prospects. Regional Studies 7, p. 239-251, 1973.

Rosen, K. T.; Resnick, M. The size distribution of cities: an examination of the Pareto law and primacy. Journal of Urban Economics 8, p. 165-186, 1980.

Shukri, I. M.; Alshuwaikhat, H. B. City-size distribution in the Saudi Arabian urban system. International Planning Studies, v. 1, issue 2, Jun 1996.

Song, S.; Zhang, K. H. Urbanisation and city size distribution in China. Urban Studies, v. 39, n. 12, p. 2317-2327, 2002.

Soo, K. T. Zipf's law for cities: a cross-country investigation. Regional Science and Urban Economics 35, p. 239-263, 2005.

Storper, M.; Walker, R. The capitalist imperative - Territory, technology, and industrial growth. USA and UK: Blackwell Publishers, 1989.

Veiga, J. E. A dimensão rural do Brasil. Estudos Sociedade e Agricultura 22, Abril 2004a. (http://www.econ.fea.usp.br/zeeli/).

. A atualidade da contradição urbano-rural. Análise territorial da Babia rural. Série Estudos e Pesquisas 71. Salvador: SEI, 2004b. (http:// www.econ.fea.usp.br/zeeli/).

. Destinos da ruralidade no processo de globalização. Estudos Avançados 51, p. 51-67, 2004c. (http://www.econ.fea.usp.br/zeeli/).

. A relação rural/urbano no desenvolvimento regional. Cadernos do CEAM 17, Fevereiro 2005. Brasília: Centro de Estudos Avançados Multidisciplinares da Universidade de Brasília - UnB (http://www.econ.fea.usp.br/zeeli/).

Wheaton, W.; Shishido, H. Urban concentration, agglomeration economies, and the level of economic development. Economic Development and Cultural Change, 1979.

World Bank. World Bank Data Bank 2003. USA, Washington: World Bank, 2003.

Endereço para contato: R. Curitiba 832 - 7ª andar - CEP 30170-120 - Brasília - DF.

E-mail:rmruiz@cedeplar.ufmg.br. Homepage: www.cedeplar.ufmg.br.

(Recebido em novembro de 2004. Aceito para publicação em julho de 2005). 